Two Birds with One Stone: Manipulating Colloids Assembled into Amorphous and Ordered Photonic Crystals and Their Combinations

\title{
for Coding-Decoding
}

Dongpeng Yang ${ }^{*}, \dagger$, , Yiwanting $\mathrm{Hu}^{\star, \S}$, Yang $\mathrm{Hu}^{\dagger}$, and Shaoming Huang ${ }^{*}, \dagger$

$\uparrow$ School of Materials and Energy, Guangdong University of Technology, Guangzhou 510006, P. R. China

†Zhejiang Key Laboratory of Carbon Materials, Wenzhou University, Wenzhou 325027, P. R. China

a)

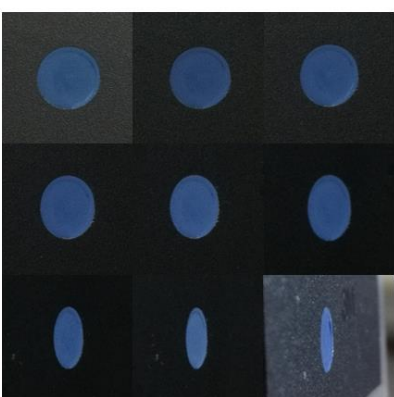

d)

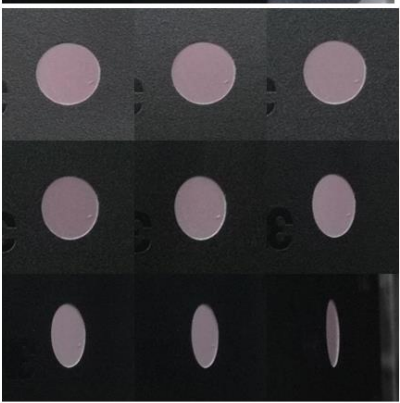

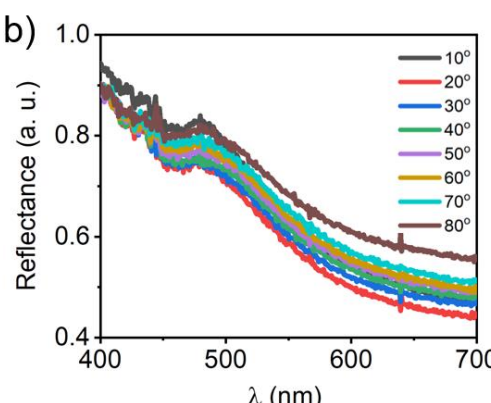

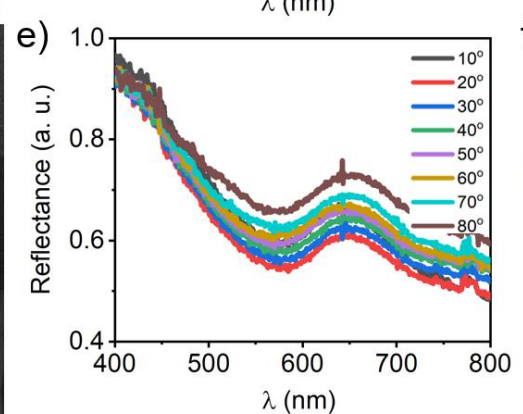

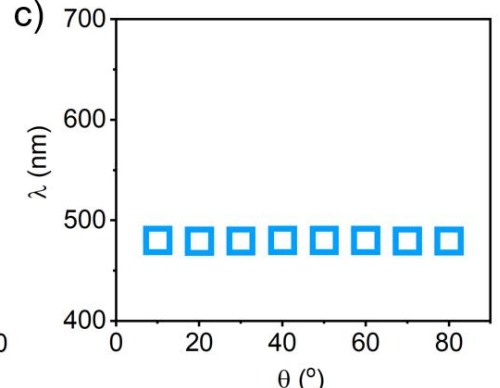

f)

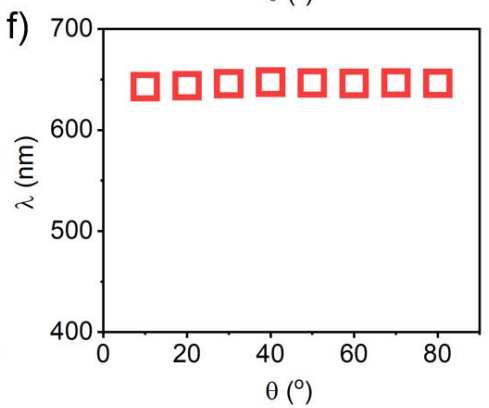

Figure S1. (a, d) Digital photos, (b, e) angle-resolved spectra, and (c, f) the corresponding plots of peak for the reflection spectra as a function of detect angle. The APCs with (a-c) blue and (d-f) red colors are assembled from the particles of 218 and $294 \mathrm{~nm}$, respectively.

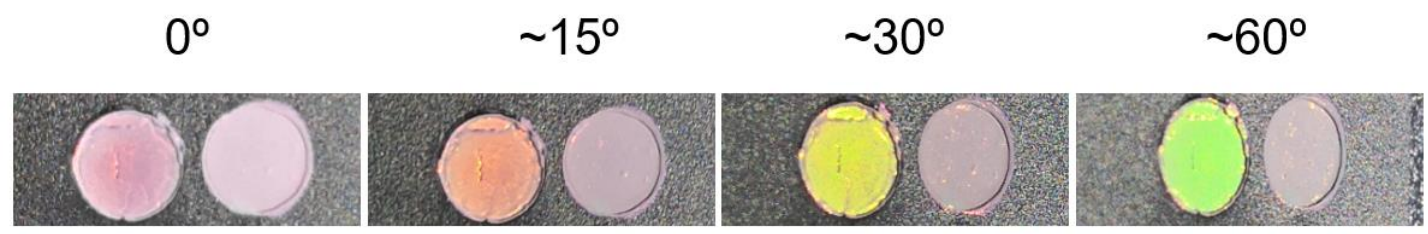

Figure S2. Digital photos of APCs and OPCs at different viewing angles, left side: OPCs, right side: APCs. 
Table S1. The general information of the photonic crystal samples used in Figure 4-6.

\begin{tabular}{|c|c|c|c|c|}
\hline Sample & $\begin{array}{l}\text { Particles } \\
\text { size }(\mathrm{nm})\end{array}$ & $\lambda(\mathrm{nm})$ & $\mathrm{APCs} / \mathrm{OPCs}$ & Reflection intensity \\
\hline \multirow{2}{*}{ Figure 4} & \multirow{2}{*}{236} & \multirow{2}{*}{522} & OPCs & $20-80$ (pH: 1-4) \\
\hline & & & APCs & 5-15 (pH: 5-6) \\
\hline \multirow{2}{*}{ Figure 5} & \multirow{2}{*}{305} & \multirow{2}{*}{668} & APCs & 6 \\
\hline & & & OPCs & 30 \\
\hline \multirow{9}{*}{ Figure 6} & 296 & 650 & OPCs & 37 (TL) \\
\hline & 236 & 522 & OPCs & 43 (TM) \\
\hline & 289 & 633 & OPCs & 46 (TR) \\
\hline & 246 & 540 & APCs & 5 (ML) \\
\hline & 324 & 710 & OPCs & $38(\mathrm{MM})$ \\
\hline & 251 & 549 & OPCs & 52 (MR) \\
\hline & 289 & 633 & OPCs & 45 (BL) \\
\hline & 263 & 576 & OPCs & $42(\mathrm{BM})$ \\
\hline & 305 & 668 & APCs & $4(\mathrm{BR})$ \\
\hline
\end{tabular}

Here, T, M, B, L, and R presents the top, middle, bottom, left and right, respectively. For example, the TL means the first number of the top layer. 